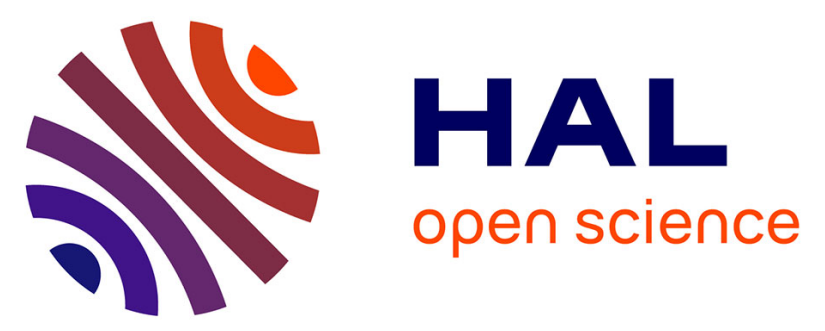

\title{
Simultaneous Registration and Change Detection in Multitemporal, Very High Resolution Remote Sensing
} Data

Maria Vakalopoulou, Konstantinos Karatzalos, Nikos Komodakis, Nikos Paragios

\section{To cite this version:}

Maria Vakalopoulou, Konstantinos Karatzalos, Nikos Komodakis, Nikos Paragios. Simultaneous Registration and Change Detection in Multitemporal, Very High Resolution Remote Sensing Data. 2015 IEEE Conference on Computer Vision and Pattern Recognition Workshops (CVPRW), Jun 2015, Boston, United States. pp.61-69, 10.1109/CVPRW.2015.7301384 . hal-01264072

\section{HAL Id: hal-01264072 \\ https://hal.science/hal-01264072}

Submitted on 16 Feb 2016

HAL is a multi-disciplinary open access archive for the deposit and dissemination of scientific research documents, whether they are published or not. The documents may come from teaching and research institutions in France or abroad, or from public or private research centers.
L'archive ouverte pluridisciplinaire $\mathbf{H A L}$, est destinée au dépôt et à la diffusion de documents scientifiques de niveau recherche, publiés ou non, émanant des établissements d'enseignement et de recherche français ou étrangers, des laboratoires publics ou privés. 


\title{
Simultaneous Registration and Change Detection in Multitemporal, Very High Resolution Remote Sensing Data
}

\author{
Maria Vakalopoulou ${ }^{1,2}$, Konstantinos Karatzalos ${ }^{1}, \quad$ Nikos Komodakis $^{2}$, Nikos Paragios ${ }^{3}$ \\ ${ }^{1}$ Remote Sensing Laboratory, Natio- \\ nal Technical University of Athens \\ mariavakemail.ntua.gr \\ ${ }^{2}$ Ecole des Ponts ParisTech, \\ University Paris-Est \\ karank@central.ntua.gr
${ }^{3}$ Center for Visual Computing Ecole Centrale de Paris
nikos.paragiosdecp. fr \\ nikos.komodakisdenpc.fr
}

\begin{abstract}
In order to exploit the currently continuous streams of massive, multi-temporal, high-resolution remote sensing datasets there is an emerging need to address efficiently the image registration and change detection challenges. To this end, in this paper we propose a modular, scalable, metric free single shot change detection/registration method. The approach exploits a decomposed interconnected graphical model formulation where registration similarity constraints are relaxed in the presence of change detection. The deformation space is discretized, while efficient linear programming and duality principles are used to optimize a joint solution space where local consistency is imposed on the deformation and the detection space. Promising results on large scale experiments demonstrate the extreme potentials of our method.
\end{abstract}

\section{Introduction}

The current generation of space-borne and airborne sensors are generating nearly continuous streams of massive multi-temporal high resolution remote sensing data. However, in order to efficiently exploit these datasets their accurate co-registration is the first indispensable processing step. Despite the fact that image registration is among the most studied problems in computer vision, most remote sensing and GIS software still employ semi-automated registration procedures when it comes to very large, multispectral, very high resolution satellite data [17, 7]. This is, however, far from a cost-effective solution especially if we consider huge multi-temporal datasets that require accurate coregistration [13, 12].

In addition, the primary goal of the analysis of multitemporal datasets is the detection of changes between different land cover types $[3,11]$. In particular, change detection

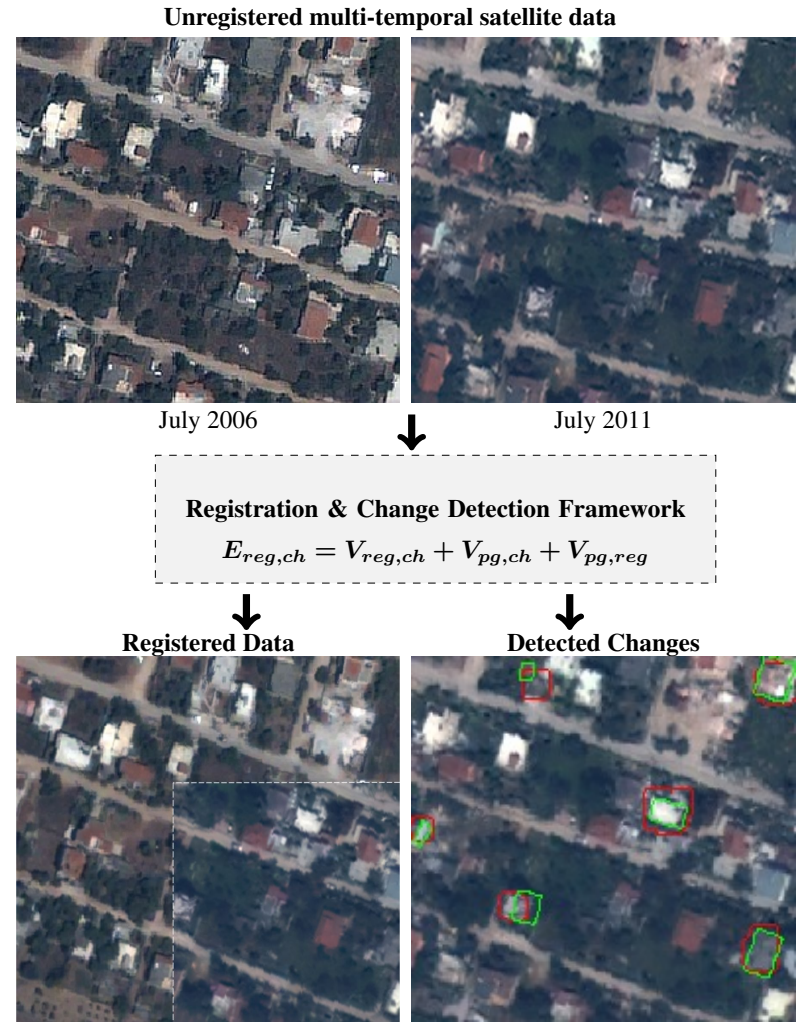

Figure 1. The developed framework addresses simultaneous the registration and change detection tasks.

of man-made objects is still an emerging challenge due to the significant importance for various engineering and environmental applications [18, 5, 10, 25, 3]. Apart from National and local government applications like the update of cadastral and other GIS databases, companies like Google and Microsoft are seeking to include extensively up-to-date 2D and 3D urban models in their products (e.g., Microsoft Virtual Earth and Google Earth). 
Change detection, however, from multi-temporal earth observation data is not a trivial task and still remains a challenge. Among the various recently proposed methods, those based on Markov Random Fields [8, 24, 1], kernels [2, 26] and neural networks [22, 23] have gained important attention. Focusing on man-made object change detection [4, 20] in urban and peri-urban regions, several approaches have been proposed based on very high resolution optical and radar data [19, 23, 6, 20]. However, these change detection techniques assume and require accurately co-registered data in order to perform pixel-by-pixel or region-by-region multi-temporal data fusion, correlation or any change analysis.

In this paper, we propose a simultaneous registration and change detection approach motivated by the fact that on one hand, the registration of very high resolution data seems to be optimally addressed through deformation grids and powerful discrete optimization [12], while on the other hand, the desired changes are located in the regions for which correspondences between the unregistered multi-temporal data can not be established (Figure 1).

To this end, we have designed, developed and evaluated a modular, scalable, metric-free single shot change detection/registration method. The approach exploits a decomposed interconnected graphical model formulation where registration similarity constraints are relaxed in the presence of change detection. We employ a discretized, gridbased deformation space. State-of-the-art linear programming and duality principles have been employed to optimize the joint solution space where local consistency is imposed on the deformation and the detection space. The unsupervised framework has been designed to handle and process large very high resolution multispectral remote sensing data, while optimized for man-made object change detection in urban and peri-urban regions. The developed method has been validated through large scale experiments on several multi-temporal very high resolution optical satellite datasets.

The main contributions of the developed method are (i) the novel, single and modular joint registration and change detection framework, (ii) the metric-free formulation which allows numerous and change-specific implementations, (iii) the low computational complexity which allows near realtime performance with gpu implementation. It should be mentioned that the detected changes can not be directly employed for the update of, e.g., a geospatial database, since the developed unsupervised framework does not include any prior information about the geometry of the man-made objects.



Figure 2. Each graph contains a smoothness term which imposes the necessary homogeneity within the graph. The interaction between the two graphs is performed by the similarity cost which connect the registration with the change detection terms.

\section{Methodology}

\subsection{MRF formulation}

We have designed and built an MRF model over two different graphs of the same dimensions. The first deformable graph corresponds to the registration term $\left(G_{r e g}\right)$ and the second one to the change detection term $\left(G_{c h}\right)$. Each graph contains a smoothness term which impose the necessary homogeneity within the graph. The interaction between the two graphs is performed by the similarity cost which connect the registration with the change detection terms.

Each graph is superimposed on the image [9] and therefore every node of the graph acts and depends on a subset of pixels in the vicinity (depending on the chosen interpolation strategy). With such a manner every pixel can participate through a certain weight to the graph related to its distance from the nodes. The computational complexity is therefore lower as graph's dimensions are smaller than the unregistered raw images. In particular, the dimensions of the graph are related to the image dimensions forming a trade off between accuracy and computational complexity. In such a setting the deformation of a pixel is defined through an interpolation of the displacement of the proximal graph nodes as follows:

$$
T(x)=x+\sum_{p \in G} \eta(\|x-p\|) d_{p}
$$

where $d_{p}$ is a displacement value, $\eta($.$) is the projection$ function, $p$ is a control point and $x$ is a pixel in the image.

After the optimization, the optimal labels will be projected to the image pixels using the same projection function $\eta($.$) . Once the similarity criterion has been defined, the$ next step consists of imposing certain continuity on the deformation space which is discussed in the next subsection.

That way, we formulate an energy function $E_{\text {reg,ch }}=$ $E_{r e g}+E_{c h}$ and we couple the two different graphs to one. 
The label for each node $p$ belonging to the graph $G$, will be $l_{p}=\left[l^{c}, l^{r e g}\right]$ where $l^{c}$ are the labels for the change detection, $l^{c} \in\{0,1\}$ and $l^{r e g}$ are the labels for the registration $l^{r e g} \in \Delta$ where $\Delta=\left[d^{1}, \ldots, d^{n}\right]$ corresponding to all possible displacements. Concluding the label space can be summarized as $L=\{0,1\} \times \Delta$.

\subsection{The Registration Energy Term}

Let us denote a pair of images where $A$ is the source image and $V$ is the target image defined on a domain $\Omega$. The goal of image registration is to define a transformation map $T$ which will project the source image to the target image.

$$
V(x)=A \circ T(x)
$$

Let us consider a discrete set of labels $L_{r e g}=[1, \ldots, n]$, and a set of discrete displacements $\Delta=\left[d^{1}, \ldots, d^{n}\right]$. We seek to assign a label $l_{p}^{r e g}$ to each grid node $p$, where each label corresponds to a discrete displacement $d^{l_{p}^{r e g}} \in \Delta$.

The energy formulation for the registration comprises of a similarity cost (that seeks to satisfy the equation 2) and a smoothness penalty on the deformation domain. The similarity cost depends on the presence of changes and will be subsequently defined. The smoothness term penalises neighbouring nodes that have different displacement labels, depending on the distance of the labelled displacements.

$$
V_{p q, r e g}\left(l_{p}^{r e g}, l_{q}^{r e g}\right)=\left\|d_{p}^{l_{p}^{r e g}}-d^{l_{q}^{r e g}}\right\|
$$

where $p$ and $q$ are neighbouring nodes.

\subsection{The Change Detection Energy Term}

The goal of the change detection term is to estimate the changed and unchanged image regions. We employ two different labels in order to address the change detection problem $l_{p}^{c} \in[0,1]$. The energy formulation for the change detection corresponds to a smoothness term which penalizes neighbouring nodes with different change labels.

$$
V_{p q, c h}\left(l_{p}^{c}, l_{q}^{c}\right)=\left\|l_{p}^{c}-l_{q}^{c}\right\|
$$

\subsection{Coupling the Energy Terms}

The coupling between change detection and registration is achieved through the interconnection between the two graphs. Assuming a pair of corresponding nodes of the two graphs, one would expect that in the absence of change the similarity cost should be satisfied and in that case the potential will be:

$$
\begin{array}{r}
V_{\text {reg }, \text { ch }}\left(l_{p}^{r e g}, l_{p}^{c}\right)=\left(1-l_{p}^{c}\right) \cdot \int_{\Omega} \hat{\eta}(\|x-p\|) \\
\rho\left(V(x), A\left(x+d^{l_{p}^{r e g}}\right)\right) d x+l_{p}^{c} \cdot C
\end{array}
$$

where we simply take all pixels in the vicinity of the graph node and project them back to the grid node with a weight that is proportional to the distance. In the presence of change, we use a fixed cost $C$. These two terms are integrated as in (equation 5) which simply uses a fixed cost in the presence of changes and the image matching cost in their absence.

With a slight abuse of notation we consider a node with an index $p \in G$ (we recall that the two graphs are identical) corresponding to the same node throughout the two graphs $\left(G_{r e g}, G_{c h}\right)$.We can now integrate all terms to a single energy which detect changes, establishes correspondences and impose smoothness in the change detection and the deformation map as follows:

$$
\begin{array}{r}
E_{\text {reg,ch }}\left(l^{c}, l^{r e g}\right)=w_{1} \cdot \sum_{p \in G} V_{r e g, c h}\left(l_{p}^{r e g}, l_{p}^{c}\right)+ \\
w_{2} \cdot \sum_{p \in G_{r e g}} \sum_{q \in N(p)} V_{p q, r e g}\left(l_{p}^{r e g}, l_{q}^{r e g}\right)+ \\
w_{3} \cdot \sum_{p \in G_{c h}} \sum_{q \in N(p)} V_{p q, c h}\left(l_{p}^{c}, l_{q}^{c}\right)
\end{array}
$$

where $V_{\text {reg,ch }}\left(l_{p}^{r e g}, l_{p}^{c}\right)$ represents the coupling term for each node at each label, $V_{p q, r e g}\left(l_{p}^{r e g}, l_{q}^{r e g}\right)$ the pairwise or binary term for the registration and $V_{p q, c h}\left(l_{p}^{c}, l_{q}^{c}\right)$ the pairwise for the change detection.

In particular, a similarity function $\rho($.$) is used in order$ to compare the two images, while a constant value $C$ is used in order to define the changes. In such a setting, optimizing an objective function seeking similarity correspondences is not meaningful and deformation vectors should be the outcome of the smoothness constraint on the displacement space. However, the areas of change are unknown and is one of the objective of the optimization process. Without loss of generality we can assume that the matching cost corresponding to change can correspond to a value that can be determined from the distribution of these costs on the entire domain (it is metric dependent). Let us consider that this value is known and that it is independent from the image displacements, so we can distinguish the regions that have been changed. The advantage of the methodology is that by solving the two problems simultaneously first we have less false changes caused by the misregistration of the images and then the changed regions do not affect the entire displacement map, as we do not calculate the displacement there and their final displacement is caused by the unchanged neighbour regions.

Finally, the pairwise costs for both terms have been described in equation 3 and 4.

\subsection{Optimization}

There are several techniques for the minimization of an MRF model which can be generally summarised into those 
based on the message passing and those on graph cut methods. The first category is related to the linear programming relaxation [14]. The optimization of the implementation is performed by FastPD which is based on the dual theorem of linear programming $[15,16]$.

\section{Implementation}

The minimization of the MRF energy is performed by a multi-scale framework. Concerning the image, iteratively different levels of Gaussian image pyramids are used. Concerning the grid, in a similar way we consider different levels of it, beginning with a sparser grid. Having very large remote sensing imagery the multi-scale approach diminishes the computational complexity without losing in terms of accuracy. The different levels of the images and the grid with the consistency of nodes in the grid are defined by the user. In all our experiments, 2 image and 3 grid levels were found adequate for the very high resolution satellite data.

Regarding the label space, a search for possible displacements along 8 directions ( $\mathrm{x}, \mathrm{y}$ and diagonal axes) is performed, while the change labels are always two and correspond to change or no change description. The number of the registration labels is the same at each level. Depending on the parameter label factor the values of registration labels change towards the optimal ones. The source image is deformed according to the optimal labels and it is updated for the next level. The value 0.8 was employed for updating the registration labels. Last but not least, the maximum displacement was set smaller than 0.4 times the distance of two consecutive nodes in order to preserve the right displacement of every node.

In addition, several methods block matching methods can be employed. Semantic changes in multitemporal imagery affect the local intensities and also change the structure of the region. One of the problems in traditional change detection techniques, is that change in intensities does not directly mean semantic change. This was crucial since the focus, here, was on urban and peri-urban regions and manmade object changes. The optimal displacement using the SADG function are calculated using the weighted sum of the difference between the pair and the gradient inner product. On the other hand any other similarity measure as mutual information, normalized cross correlation, ratio correlation can be used. In Section 4 we have tested different similarity functions. For the SADG metric and focused on man-made object changes, the fixed cost $C$ was set to 100 . In particular, higher $C$ values result to less changes. The parameter is not so sensitive since values between 90 to 120 lead to comparable results.

Last but not least, the number of iterations per level was set to 10 , the regularization parameter for the registration task to 35 and for the change detection one to 3.5. The function used for the projection from pixels to nodes and reverse was the Cubic B-splines.

\section{Experimental Results and Evaluation}

\subsection{Dataset}

The developed framework was applied to several pairs of multispectal VHR images from different satellite sensors (i.e., Quickbird and WorldView-2). The multi-temporal dataset covers approximately a $9 \mathrm{~km}^{2}$ region in the East Prefecture of Attica in Greece. All datasets were acquired between the years of 2006 and 2011. The dataset is quite challenging both due to its size and the pictured complexity derived from the different acquisition angles. For the quantitative evaluation the ground truth was manually collected and annotated after an attentive and laborious photointerpretation done by an expert.

\subsection{Experimental Results}

Extensive experiments were performed over several images pairs and based on several similarity metrics namely the Sum of Absolute Differences (SAD), the Sum of Square Differences (SSD), the Normalized Cross Correlation (NCC), the Normalized Mutual Information (NMI), the Correlation Ratio (CR), the Sum of Gradient Inner Products (GRAD), the Normalized Correlation Coefficient plus Sum of Gradient Inner Products (CCGIP), the Hellinger Distance (HD), the Jensen-Renyi Divergence (JRD), the Mutual Information (MI) and the Sum Absolute of Differences plus Gradient Inner Products (SADG). The experimental results were evaluated both qualitative and quantitative for the registration and the change detection tasks.

Moreover, in order to evaluate quantitatively the developed algorithm, the standard quality metrics of Completeness, Correctness and Quality were calculated on the detected object level. The True Positives (TP), False Negatives (FN) and False Positives (FP) were calculated in all cases.

$$
\begin{gathered}
\text { Completeness }=\frac{T P}{T P+F N} \\
\text { Correctness }=\frac{T P}{T P+F P} \\
\text { Quality }=\frac{T P}{T P+F P+F N}
\end{gathered}
$$

where TP is the number of correctly detected changes, FN is the number of changes that haven't been detected by the algorithm and FP is the number of false alarms.

Regarding the evaluation of the registration, a number of ground control points (GCPs) were manually collected in both unregistered and registered data. In particular, the GCPs contained several points on building roof tops which usually have the largest displacements. The displacement 

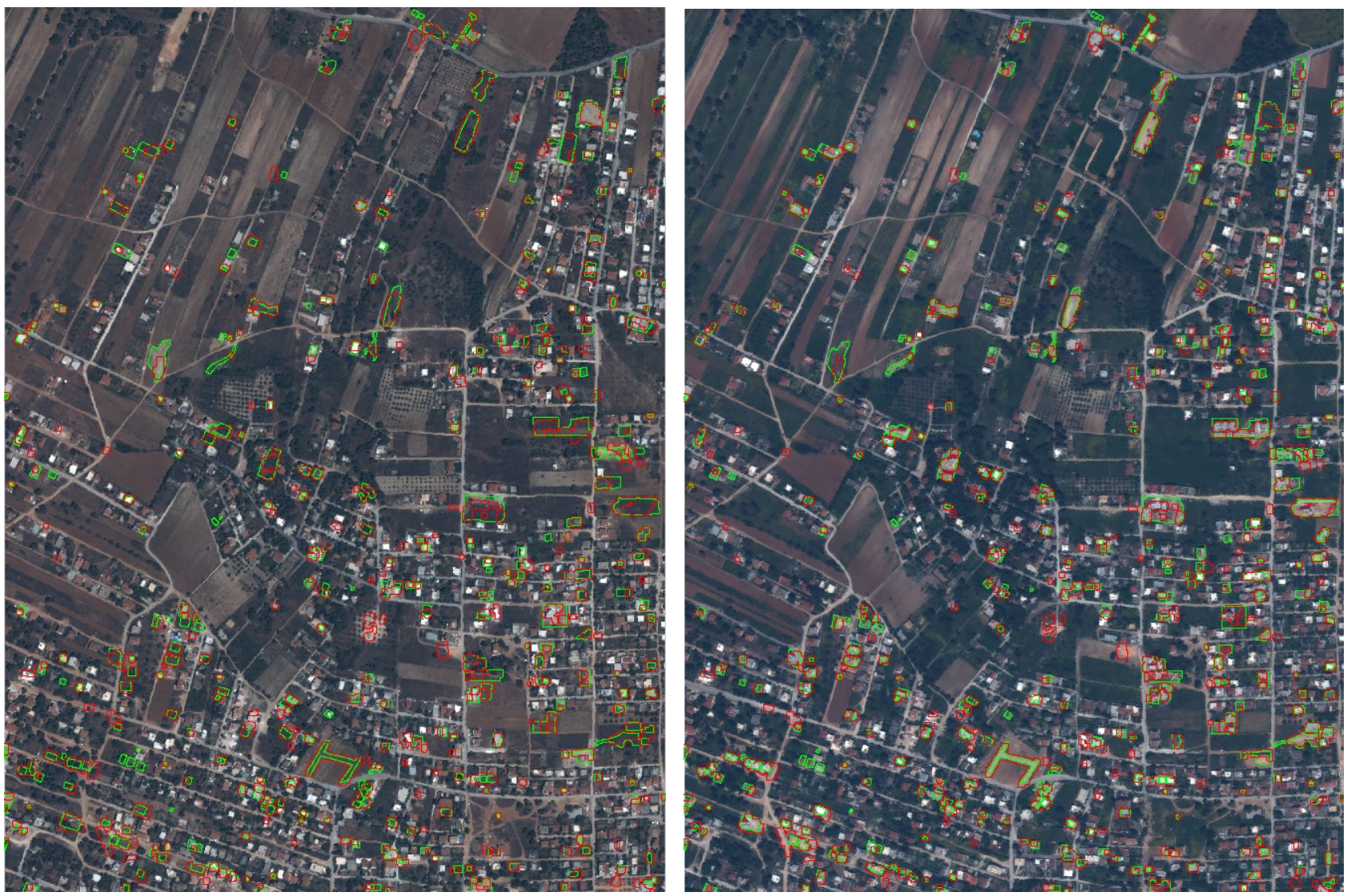

Figure 3. Experimental results after the application of the developed method. The detected changes are shown with red color and the ground truth with green. Both are superimposed to the very high resolution satellite images acquired in 2006 (left) and 2011 (right).

errors before and after the application of the developed framework were calculated.

In Table 1 the mean displacement errors (in pixels) for both axes $(x, y)$ and the mean distance before and after the algorithm's convergence are presented. In particular, the table contains the registration results for different similarity metrics. It can be observed that the framework acts quite robustly regarding the registration procedure since in all cases regardless of the employed similarity metric the mean displacement was lower than 4 pixels. In accordance with the literature the NCC metric performs optimally when optical data are considered [12].

Regarding the evaluation for the man-made change detection task, experimental results after the application of the developed method are shown in Figure 3 and Figure 4. In particular, in Figure 3 the detected changes are shown with a red color while the ground truth polygons are shown with green. It can be observed that in most cases the detected changes are in accordance with the ground truth. There are sporadically a few FN and FP due to, in most cases, high spectral variations between soil, gravel and other construction materials between the two acquisition periods. In all cases, the experimental results were derived based on the SADG similarity measure and parameters tuned for detecting changes in man-made objects, as described in Section 3.

\begin{tabular}{|c|c|c|c|}
\hline & Dx (pixels) & Dy (pixels) & DS (pixels) \\
\hline \hline Before & 7.61 & 7.31 & 11.04 \\
\hline \hline SADG & 2.45 & 2.03 & 3.18 \\
SAD & 2.57 & 1.32 & 3.05 \\
SSD & 3.12 & 2.04 & 4.02 \\
NCC & $\mathbf{2 . 2 3}$ & $\mathbf{1 . 3 3}$ & $\mathbf{2 . 6 0}$ \\
NMI & 2.53 & 1.92 & 2.92 \\
CR & 2.67 & 1.04 & 3.01 \\
GRAD & 3.23 & 1.74 & 3.7 \\
CCGIP & 2.84 & 2.5 & 4.13 \\
JRD & 2.34 & 1.34 & 2.73 \\
HD & 2.42 & 1.08 & 2.79 \\
MI & 2.76 & 1.02 & 2.95 \\
\hline
\end{tabular}

Table 1. Quantitative evaluation after the application of the developed framework under several similarity metrics. The calculated mean displacement errors are shown. 
Case\#1

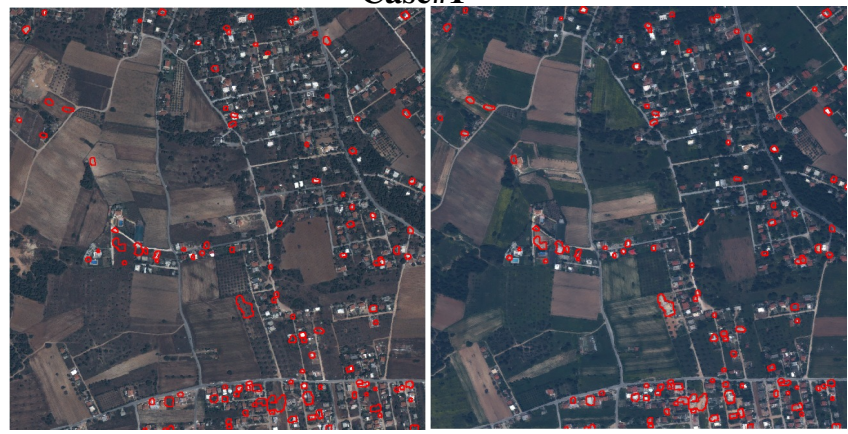

Case\#3



Case\#5

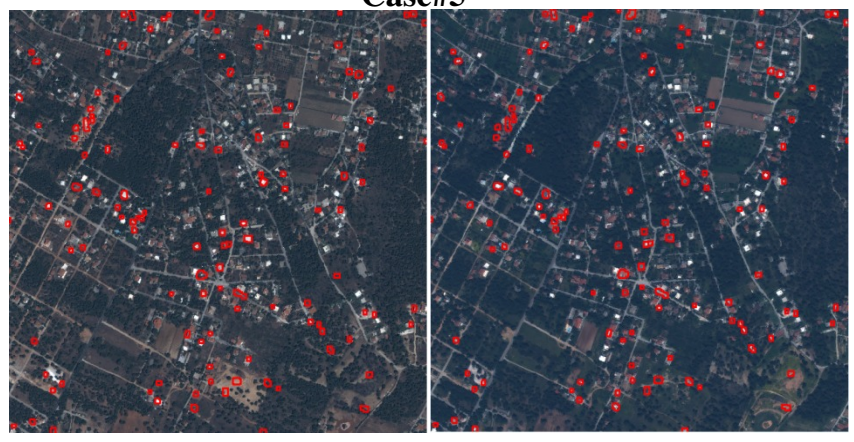

Case\#7

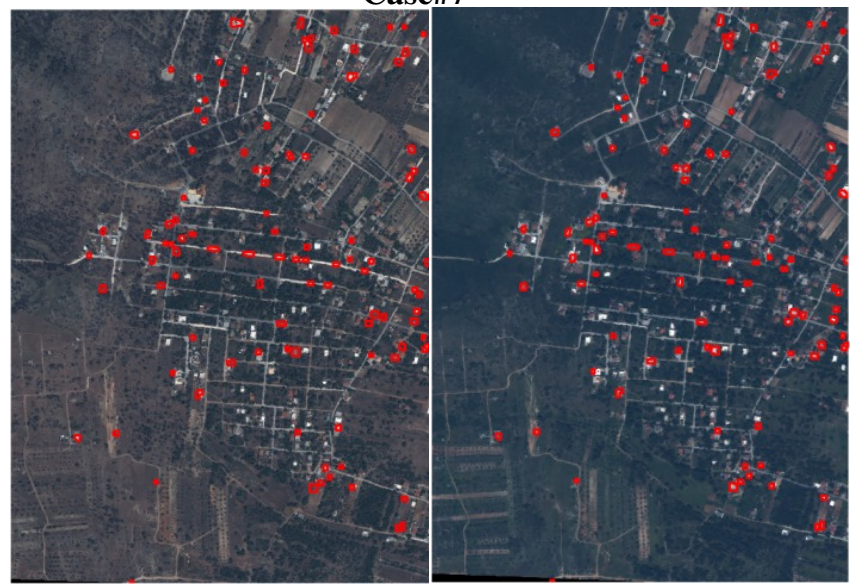

Case\#2

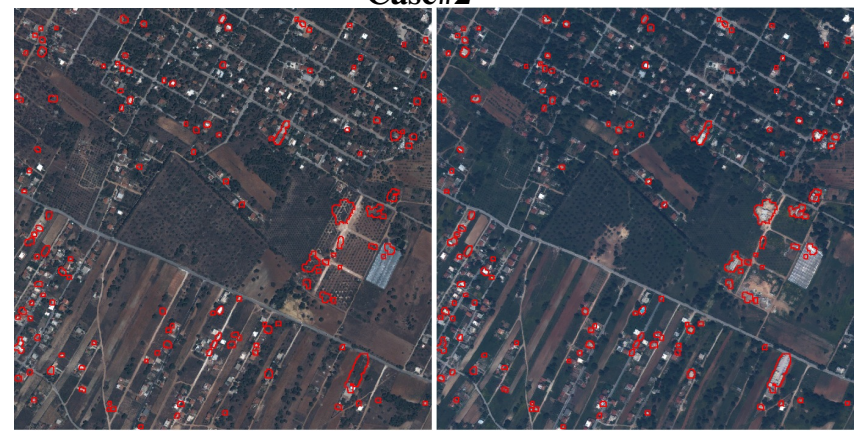

Case\#4

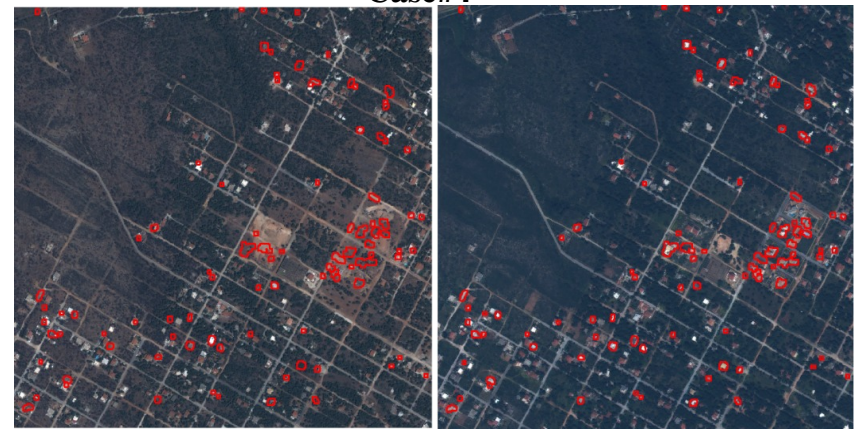

\section{Case\#6}



Case\#8

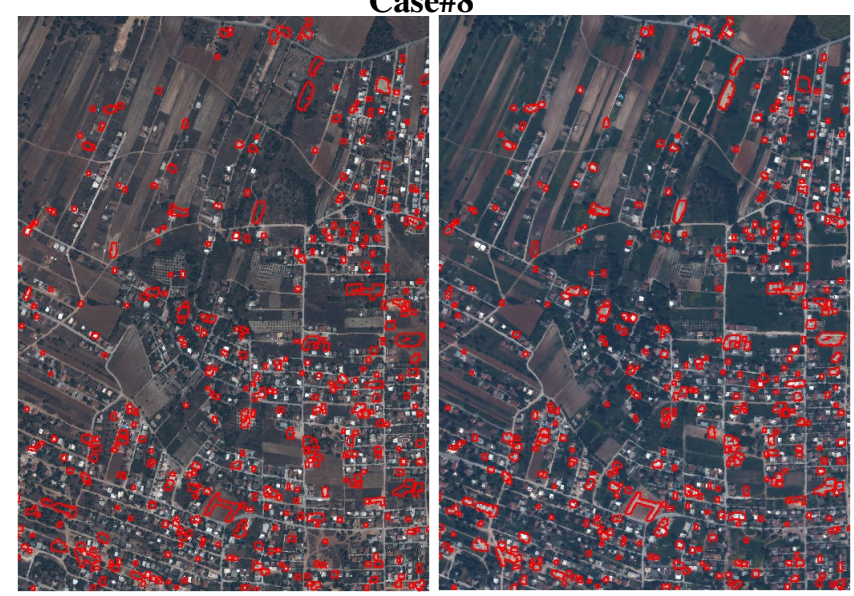

Figure 4. Experimental results after the application of the developed method. The detected changes are shown with red color and are superimposed to the multi-temporal very high resolution satellite images. 


\begin{tabular}{|c|c|c|c|}
\hline Method & Complet \% & Corr \% & Quality \% \\
\hline \hline IRMAD & 67.2 & 36.8 & 30.1 \\
\hline \hline SADG & 92.2 & $\mathbf{8 0 . 1}$ & $\mathbf{7 4 . 4}$ \\
SAD & $\mathbf{9 5 . 2}$ & 64.9 & 60.01 \\
SSD & 94.1 & 67.3 & 61.4 \\
NCC & 77.7 & 40.5 & 34.8 \\
NMI & 55.3 & 62.8 & 50.1 \\
CR & 60.5 & 30.3 & 25.2 \\
GRAD & 35.1 & 49.3 & 23.1 \\
CCGIP & 77.8 & 40.4 & 34.9 \\
JRD & 39.6 & 50.1 & 30.4 \\
HD & 83.6 & 60.1 & 57.8 \\
MI & 41.9 & 52.8 & 30.1 \\
\hline
\end{tabular}

Table 2. Quantitative evaluation for the change detection task after the application of the developed method. The standard measures of detection completeness and correctness on object level have been calculated for different similarity measures.

In Figure 4 experimental results from several cases are presented. The detected changes are shown with a red color in both acquisition dates/images. It can be observed that the framework can detect changes related to man-made objects based both on the spectral variation i.e., absolute difference and geometric features, inner gradient products.

In Table 2 results from the performed quantitative evaluation are presented for the man-made change detection task. Experimental results from several similarity measures have been calculated after the application of the developed method. Results have been also compared with the unsupervised IRMAD [21] change detection algorithm. It can be observed that the SADG metric has the highest performance (overall quality) even if it doesn't score the maximum completeness rates. The behaviour of the developed method can be further observed in Figure 5, where certain examples with True Positives, False Negatives and False Positives cases are presented. The performed extensive experiments and the acquired quite promising results demonstrate the extreme potentials of the developed method in detecting changes related to man-made objects.

Last but not least, the computational efficiency of the proposed framework was evaluated. In particular, in Ta-

\begin{tabular}{|c|c|c|}
\hline \multicolumn{3}{|c|}{ Computational Performance } \\
\hline Size in $\mathrm{km}^{2}$ & Size in pixels & Time (min) \\
\hline \hline 0.25 & $1000 \times 1000$ & 6.25 \\
\hline 0.5 & $1500 \times 1300$ & 20.5 \\
\hline 1 & $2000 \times 2000$ & 27.7 \\
\hline 1.5 & $2800 \times 2140$ & 53.3 \\
\hline
\end{tabular}

Table 3. Quantitative evaluation of the computational performance.

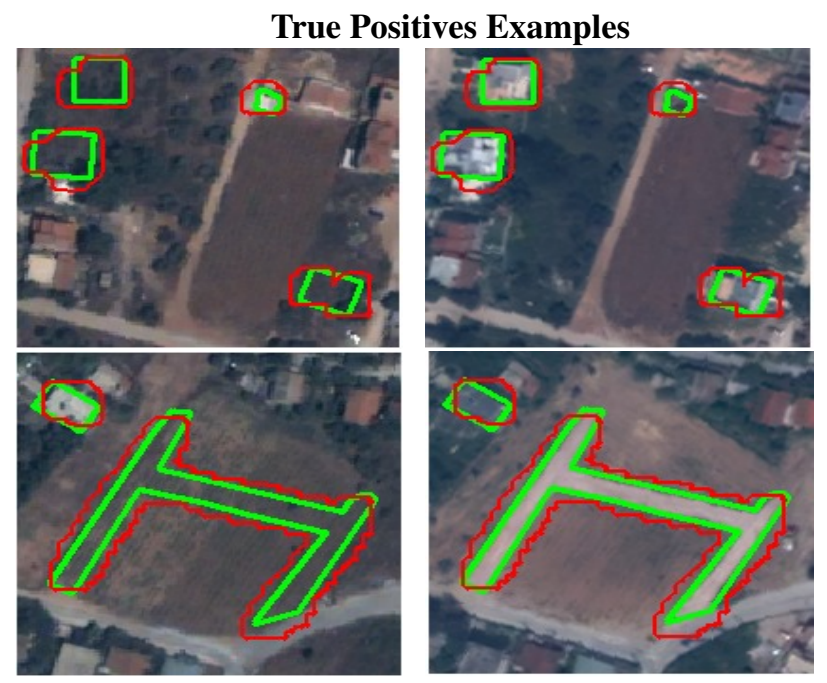

False Negatives Examples

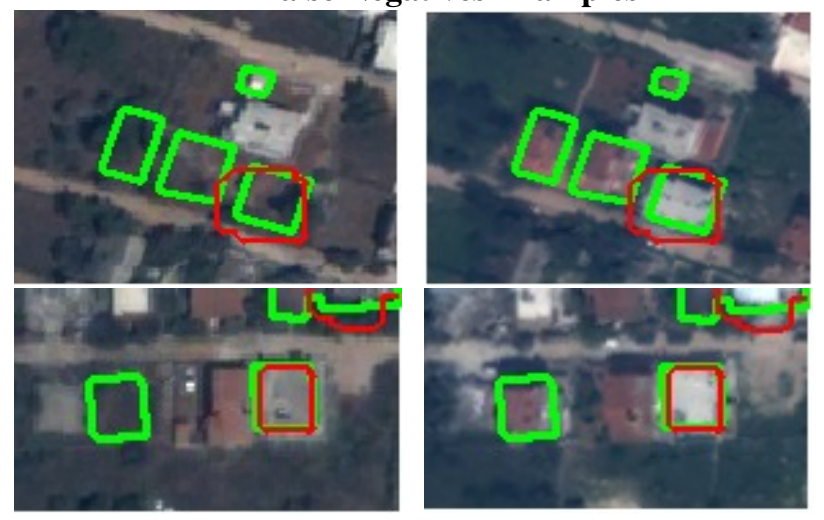

False Positives Examples

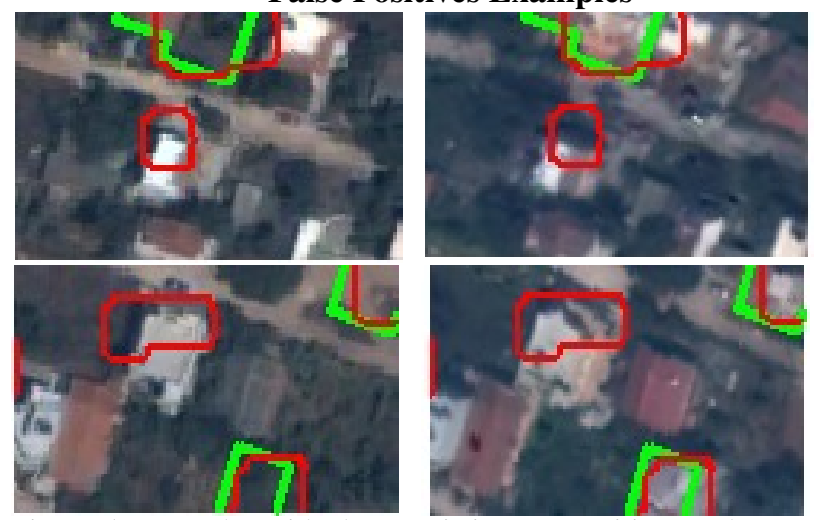

Figure 5. Examples with characteristic True Positives, False Negatives and False Positives that demonstrate the behaviour of the developed method.

ble 3 the computational time required for different image sizes with the SADG metric are presented. It should be also mentioned that the computational time is also depending on the selected set of parameters which specify, e.g., the num- 
ber of nodes in the grid, the number of labels or the number of iterations per level. The selected metric affects, also, the computational time significantly. In accordance with the literature in all our experiments the SAD, SSD, NCC and SADG were the quickest ones, while the JRD and MI were more time consuming.

\section{Conclusions}

In this paper we designed, developed and validated a novel framework which address concurrently the registration and change detection tasks in very high resolution multispectral multitemporal optical satellite data. The developed method is modular, scalable and metric free. The formulation exploits a decomposed interconnected graphical model formulation where registration similarity constraints are relaxed in the presence of change detection. The framework was optimized for the detection of changes related to man-made objects in urban and peri-urban environments. The performed large scale experiments and the acquired quite promising results demonstrate the extreme potentials of the developed method. The integration of prior knowledge regarding texture and geometric features is currently under development and a gpu implementation is among the future perspectives as well.

\section{Acknowledgement}

This research has been co-financed by the European Union (European Social Fund-ESF) and Greek national funds through the Operational Program Education and Lifelong Learning of the National Strategic Reference Framework (NSRF)-Research Funding Program THALES: Reinforcement of the interdisciplinary and/or inter-institutional research and innovation.

\section{References}

[1] C. Benedek, M. Shadaydeh, Z. Kato, T. Szirányi, and J. Zerubia. Multilayer Markov Random Field Models for Change Detection in Optical Remote Sensing Images. ISPRS Journal of Photogrammetry and Remote Sensing, page 18, 2015. 2

[2] G. Camps-Valls and L. Bruzzone. Kernel Methods for Remote Sensing Data Analysis. Wiley, 2009. 2

[3] M. J. Canty. Image Analysis, Classification and Change Detection in Remote Sensing: With Algorithms for ENVI/IDL and Python. CRC Press, 2014. 1

[4] N. Champion, D. Boldo, M. Pierrot-Deseilligny, and G. Stamon. 2D building change detection from high resolution satelliteimagery: A two-step hierarchical method based on 3d invariant primitives. Pattern Recognition Letters, 31(10):1138-1147, 2010. 2

[5] G. Doxani, K. Karantzalos, and M. Tsakiri-Strati. Monitoring urban changes based on scale-space filtering and object-oriented classification. International Journal of Ap- plied Earth Observation and Geoinformation, 15(0):38 - 48, 2012. 1

[6] N. Falco, M. Mura, F. Bovolo, J. Benediktsson, and L. Bruzzone. Change detection in vhr images based on morphological attribute profiles. Geoscience and Remote Sensing Letters, IEEE, 10(3):636-640, May 2013. 2

[7] F. Florence Tupin, J. Inglada, and J.-M. Nicolas. Remote Sensing Imagery. Wiley, 2014. 1

[8] A. Ghosh, B. Subudhi, and L. Bruzzone. Integration of gibbs markov random field and hopfield-type neural networks for unsupervised change detection in remotely sensed multitemporal images. Image Processing, IEEE Transactions on, 22(8):3087-3096, Aug 2013. 2

[9] B. Glocker, A. Sotiras, N. Komodakis, and N. Paragios. Deformable Medical Image Registration: Setting the State of the Art with Discrete Methods. Annual Review of Biomedical Engineering, 13:219-244, 2011. 2

[10] M. Hussain, D. Chen, A. Cheng, H. Wei, and D. Stanley. Change detection from remotely sensed images: From pixelbased to object-based approaches. ISPRS Journal of Photogrammetry and Remote Sensing, 80(0):91 - 106, 2013. 1

[11] K. Karantzalos. Recent Advances on 2D and 3D Change Detection in Urban Environments from Remote Sensing Data. In M. Helbich, J. Jokar Arsanjani, and M. Leitner, editors, Computational Approaches for Urban Environments, Geotechnologies and the Environment, pages 237272. 2015. 1

[12] K. Karantzalos, A. Sotiras, and N. Paragios. Efficient and automated multi-modal satellite data registration through mrfs and linear programming. IEEE Computer Vision and Pattern Recognition Workshops, pages 1-8, June 2014. 1, 2, 5

[13] M. Klaric, B. Claywell, G. Scott, N. Hudson, O. Sjahputera, Y. Li, S. Barratt, J. Keller, and C. Davis. GeoCDX: An Automated Change Detection and Exploitation System for HighResolution Satellite Imagery. Geoscience and Remote Sensing, IEEE Transactions on, 51(4):2067-2086, April 2013. 1

[14] N. Komodakis, N. Paragios, and G. Tziritas. Mrf energy minimization and beyond via dual decomposition. Pattern Analysis and Machine Intelligence, IEEE Transactions on, 33(3):531-552, March 2011. 4

[15] N. Komodakis and G. Tziritas. Approximate labeling via graph cuts based on linear programming. Pattern Analysis and Machine Intelligence, IEEE Transactions on, 29(8):1436-1453, Aug 2007. 4

[16] N. Komodakis, G. Tziritas, and N. Paragios. Performance vs computational efficiency for optimizing single and dynamic mrfs: Setting the state of the art with primal-dual strategies. Comput. Vis. Image Underst., 112(1):14-29, Oct. 2008. 4

[17] J. Le Moigne, N. S. Netanyahu, and R. D. Eastman. Image Registration for Remote Sensing. Cambridge University Press, 2011. 1

[18] N. Longbotham, F. Pacifici, T. Glenn, A. Zare, M. Volpi, D. Tuia, E. Christophe, J. Michel, J. Inglada, J. Chanussot, and Q. Du. Multi-modal change detection, application to the detection of flooded areas: Outcome of the 2009-2010 data fusion contest. Selected Topics in Applied Earth Observations and Remote Sensing, IEEE Journal of, 5(1):331-342, Feb 2012. 1 
[19] S. Marchesi, F. Bovolo, and L. Bruzzone. A context-sensitive technique robust to registration noise for change detection in vhr multispectral images. Image Processing, IEEE Transactions on, 19(7):1877-1889, July 2010. 2

[20] C. Marin, F. Bovolo, and L. Bruzzone. Building change detection in multitemporal very high resolution sar images. Geoscience and Remote Sensing, IEEE Transactions on, 53(5):2664-2682, May 2015. 2

[21] A. Nielsen. The regularized iteratively reweighted mad method for change detection in multi- and hyperspectral data. Image Processing, IEEE Transactions on, 16(2):463478, Feb 2007. 7

[22] F. Pacifici and F. Del Frate. Automatic change detection in very high resolution images with pulse-coupled neural networks. Geoscience and Remote Sensing Letters, IEEE, 7(1):58-62, Jan 2010. 2

[23] C. Pratola, F. Del Frate, G. Schiavon, and D. Solimini. Toward fully automatic detection of changes in suburban areas from vhr sar images by combining multiple neural-network models. Geoscience and Remote Sensing, IEEE Transactions on, 51(4):2055-2066, April 2013. 2

[24] P. Singh, Z. Kato, and J. Zerubia. A multilayer markovian model for change detection in aerial image pairs with large time differences. In Pattern Recognition (ICPR), 2014 22nd International Conference on, pages 924-929, Aug 2014. 2

[25] A. Taneja, L. Ballan, and M. Pollefeys. City-Scale Change Detection in Cadastral 3D Models Using Images. In Computer Vision and Pattern Recognition (CVPR), 2013 IEEE Conference on, pages 113-120, June 2013. 1

[26] M. Volpi, D. Tuia, G. Camps-Valls, and M. Kanevski. Unsupervised change detection with kernels. Geoscience and Remote Sensing Letters, IEEE, 9(6):1026-1030, Nov 2012. 2 